CYSTIC FIBROSIS

\title{
Objective measurement of cough during pulmonary exacerbations in adults with cystic fibrosis
}

\author{
J A Smith, E C Owen, A M Jones, M E Dodd, A K Webb, A Woodcock

See end of article for authors' affiliations

.....................

Correspondence to: Dr J A Smith, North West Lung Research Centre, Wythenshawe Hospital Southmoor Road,

Wythenshawe,

Manchester M23 9LT, UK Jacky.Smith@manchester. co.uk

Received 17 August 2005 Accepted 24 January 2006 Published Online First 31 January 2006

\begin{abstract}
Background: Little is known about cough frequency in adults with cystic fibrosis (CF). This study aimed to determine (1) daytime and overnight cough rates in patients with CF at the beginning and end of a course of antibiotics for treatment of an exacerbation; (2) the relationship between cough frequencies and standard clinical measures of disease; and (3) the relationship between objective cough rates and the subjective assessment of cough.

Methods: Nineteen adult patients admitted with a pulmonary exacerbation performed daytime and overnight sound recordings on admission; 13 had repeat recordings prior to discharge. Coughs were manually quantified in cough seconds (time spent coughing). Patients subjectively scored their cough using a visual analogue scale (VAS) and numerical score. Lung function, C-reactive protein (CRP) levels, and sputum weights were recorded.

Results: Cough rates fell substantially with treatment; median fall in cough rate was $51.3 \%$ (IQR 32.377.5) $(p<0.001)$ for daytime and 72.2\% (28.6-90.1) $(p=0.049)$ for overnight. Multivariate regression analyses showed that forced expiratory volume in 1 second and CRP levels predicted overnight cough rates on admission. On discharge, sputum volume predicted daytime cough rates. Only the change in overnight VAS correlated with the change in objective cough rates.

Conclusions: The cough rate significantly decreases with treatment of a pulmonary exacerbation in adults with CF. Lung function, sputum volume, and CRP influences the cough rate, with the effects differing from day to night and between admission and discharge. Subjective reporting of a nocturnal cough may indicate a pulmonary exacerbation of CF in adults.
\end{abstract}

$\mathrm{P}$ ulmonary exacerbations are frequently used as an outcome measure in clinical trials of cystic fibrosis (CF). ${ }^{1}$ Exacerbations are defined based upon clinical criteria, usually an acute worsening in respiratory function and symptoms (such as a change in sputum production and an increased cough) which resolve with intensive treatment. ${ }^{2}{ }^{3}$

Because of the difficulties in measuring cough, little is known about the frequency of coughing in $\mathrm{CF}$, either in stable disease or the changes associated with an exacerbation. However, a study of cough in children with CF surprisingly did not find any difference in cough epochs with treatment of a pulmonary exacerbation. ${ }^{4}$

The causes of cough in CF are likely to be multiple, including airway inflammation, mucus, airway instability, sinus disease, and perhaps also gastro-oesophageal reflux. The relative contributions of these factors remain unknown.

We hypothesised that inpatient treatment for a pulmonary exacerbation of CF would reduce airway inflammation and sputum production and consequently coughing would be reduced. The primary outcome measure was the objective monitoring of cough before and after treatment. We have quantified cough as the time spent coughing per hour. If coughing occurs in prolonged bursts, this measure takes account of the length of these bursts as well as the number.

Secondary outcome measures were spirometry, C-reactive protein (CRP) levels, and sputum weight. Based on previous work, we predicted that these clinical markers of an exacerbation would be related to time spent coughing. ${ }^{5}$ We also examined the relationships between subjective estimates of cough severity and cough frequency.

\section{METHODS}

Subjects

Subjects were recruited from consecutive admissions with a pulmonary exacerbation of CF. Clinical parameters were used to define a CF exacerbation including change in sputum load and colour, increased breathlessness, cough and wheeze, and a fall in forced expiratory volume in 1 second $\left(\mathrm{FEV}_{1}\right)$ of $>10 \%$ compared with baseline. Subjects under 16 years of age, current smokers (three were ex-smokers), and subjects requiring high flow oxygen or non-invasive ventilation were excluded. Those infected with Burkholderia cepacia, methicillin resistant Staphylococcus aureus, and multidrug resistant Pseudomonas species were also excluded because of the potential risk of infection transfer via the cough recording equipment. The study was approved by the local ethics committee and informed written consent was obtained from all subjects.

All patients received standard treatment for their pulmonary exacerbation including intravenous antibiotics, chest physiotherapy, and an overnight intravenous aminophylline infusion starting on the day of admission. Measurement of spirometric parameters, sputum weight, and CRP was performed as part of routine monitoring.

\section{Study procedures}

Each patient had ambulatory daytime and overnight sound recordings on the day after admission and again prior to discharge. On each study day the daytime recording commenced at approximately 10.00 hours and the overnight recording at 22.00 hours; each was 10 hours in length. Sound recordings were obtained by using digital recording devices (Nomad Jukebox 3, Creative Technology Ltd, Singapore) and a lapel microphone (AOI, ECM-1025) positioned $30 \mathrm{~cm}$ from the mouth. The digital files were transferred to a personal computer for analysis and archiving. Custom written software was used to compress the sound files (remove silence

Abbreviations: CF, cystic fibrosis; CRP, C-reactive protein; $\mathrm{FEV}_{1}$, forced expiratory volume in 1 second 
and low amplitude sounds) and to assist manual counting of the time spent coughing. Time spent coughing was defined as the numbers of seconds per hour that contained at least one explosive cough sound. Coughing induced by physiotherapy was excluded from the analyses. Each recording takes approximately $2.5-3$ hours to process. We have previously validated this methodology by comparing coughs counted from digital sound recordings with those counted from video recordings and found excellent agreement between the two methods. ${ }^{6}$ To check reliability of the manual counting, 10 randomly selected tracks were recounted by a second trained observer. To examine the relationships between time spent coughing (cough seconds, cs) and cough epochs, the first 3 hours of each daytime recording was recounted in cough epochs. Cough epochs are defined as a period of coughing with less than 2 seconds interruption. ${ }^{4-10}$

Sputum was collected during the day and night and weighed at the end of each recording period. Clinical assessment routinely includes sputum collection, spirometric tests, and measurement of CRP levels (normal $<5 \mathrm{mg} / \mathrm{l}$ ).

Patients were asked to score the severity of their cough subjectively by day and by night. Two methods of subjective assessment were used; a visual analogue scale (100 mm line marked "no cough" to "worst cough") and a cough score chart with a range of scores from 0 to 5 (table 1)..$^{4}$

\section{Statistical analysis}

A power calculation to determine sample size could not be performed due to the lack of existing data on time spent coughing in CF; after consultation with a statistician it was decided to recruit 20 subjects. Both day and night cough data were positively skewed. For clarity, the median and interquartile ranges are reported for day and night, on admission and discharge. However, for the primary outcome (the comparison of time spent coughing before and after treatment) the $\log _{10}$ transforms were used and means compared using a paired $t$ test (two sided).

For the secondary hypotheses, multivariate linear regression analyses were performed to examine the influence of $\mathrm{FEV}_{1}, \mathrm{CRP}$, and sputum weight on cough frequency. Again data were $\log$ transformed for these analyses. Subjective measures of cough were also skewed but could not be improved by transformation and hence Spearman's correlations were used to examine the relationships between subjective and objective measures of cough.

Statistical analysis was performed using SPSS Version 11.0 (SPSS Inc, Chicago, IL, USA) and Prism 4.0 (Graphpad Software Inc, San Diego, CA, USA).

\begin{tabular}{|c|c|c|}
\hline Score & Daytime & Night time \\
\hline 0 & No cough during the day & No cough during the night \\
\hline 1 & Cough for one short period & Cough on waking only \\
\hline 2 & $\begin{array}{l}\text { Cough for more than two } \\
\text { short periods }\end{array}$ & $\begin{array}{l}\text { Wake once or early due to } \\
\text { cough }\end{array}$ \\
\hline 3 & $\begin{array}{l}\text { Frequent coughing which did } \\
\text { not interfere with usual } \\
\text { daytime activities }\end{array}$ & Frequent waking due to coughs \\
\hline 4 & $\begin{array}{l}\text { Frequent coughing which did } \\
\text { interfere with usual daytime } \\
\text { activities }\end{array}$ & $\begin{array}{l}\text { Frequent coughs most of the } \\
\text { night }\end{array}$ \\
\hline 5 & $\begin{array}{l}\text { Distressing cough most of the } \\
\text { day }\end{array}$ & $\begin{array}{l}\text { Distressing cough most of the } \\
\text { night }\end{array}$ \\
\hline
\end{tabular}
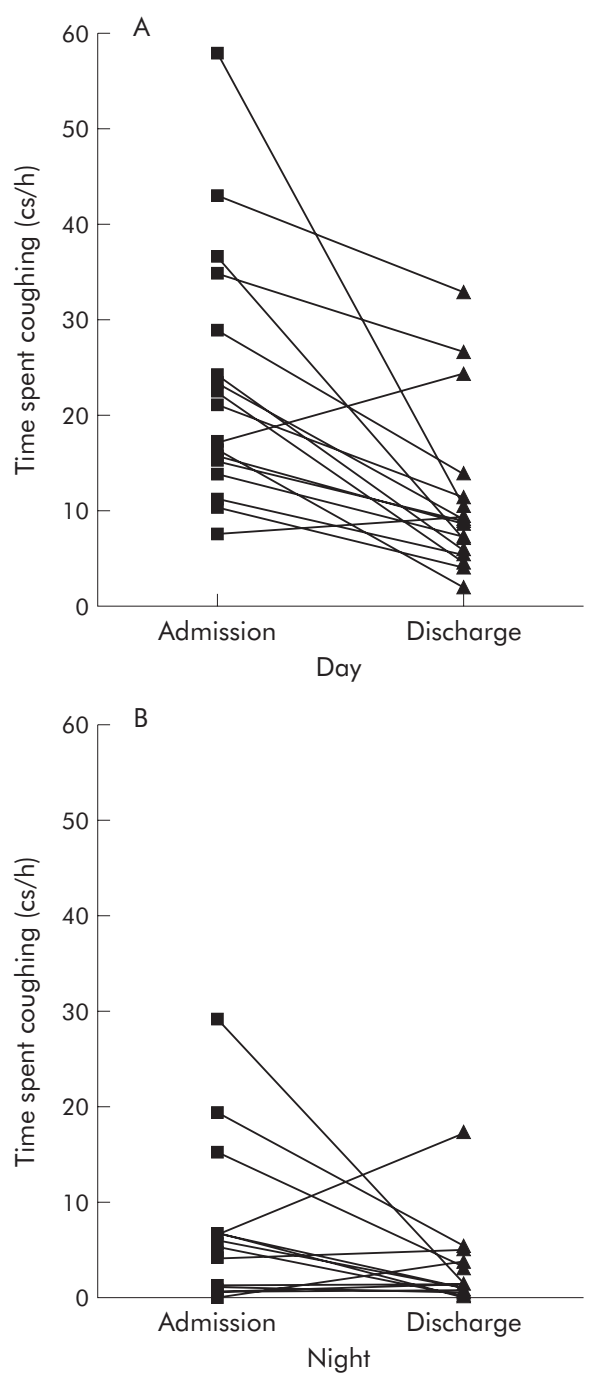

Figure 1 Time spent coughing with a pulmonary exacerbation on admission and before discharge: (A) daytime, (B) overnight.

\section{RESULTS}

Twenty patients with CF and an exacerbation of their chest disease entered the study. The median age was 23 years (interquartile range (IQR) 19-30), nine were men, and the median body mass index was $19.6 \mathrm{~kg} / \mathrm{m}^{2}$ (IQR 18.2-22.3). After the study was completed, one patient was found to be a current smoker and so was excluded. Of the remaining 19 subjects, one patient performed recordings only on admission and withdrew from further study for personal reasons. Of the 74 recordings performed, five were technically faulty; hence, cough recording data were available for 19 subjects on admission and for 13 subjects prior to discharge. Each analysis was performed on the fullest data set available. The mean (SD) time between admission and discharge recordings was 8.0 (3.0) days. Agreement between manual cough counts for two trained observers was excellent with a mean difference of 0.25 cs between observers (95\% limits of agreement -2.17 and 2.67). ${ }^{11}$

\section{Daytime and overnight cough during and after exacerbation}

After treatment there was a significant fall in time spent coughing for both day and night recording periods, with a median fall in cough rate of $51.3 \%$ (IQR 32.3-77.5) for 


\begin{tabular}{|c|c|c|c|}
\hline & Admission & Discharge & p value \\
\hline $\mathrm{FEV}_{1}$ (\% predicted) & $41.8(32-67.8)$ & $56.1(40.2-76.8)$ & 0.002 \\
\hline $\mathrm{FEV}_{1} / \mathrm{FVC}(\%)$ & $60.5(54.2-73.6)$ & $65.0(58.2-75.3)$ & 0.213 \\
\hline $\mathrm{CRP}(\mathrm{mg} / \mathrm{dl})$ & $16(1-31)$ & $5(1-13)$ & 0.050 \\
\hline \multicolumn{4}{|l|}{ Cough rate (cs/h) } \\
\hline Day & $21.2(14.0-34.9)$ & $9.0(5.8-12.8)$ & $<0.001$ \\
\hline Night & $4.8(1.0-6.8)$ & $1.5(0.8-5.0)$ & 0.049 \\
\hline \multicolumn{4}{|l|}{ Sputum (g) } \\
\hline Day & $7.1(3.8-21.8)$ & $2.8(0.0-4.5)$ & 0.041 \\
\hline Night & $2.2(0.0-9.3)$ & $0.0(0.0-4.5)$ & 0.959 \\
\hline \multicolumn{4}{|l|}{ Cough score } \\
\hline Day & $3.0(2.0-3.0)$ & $1.5(1.0-2.8)$ & 0.002 \\
\hline Night & $1.0(0.0-2.0)$ & $1.0(0.0-1.0)$ & 0.216 \\
\hline \multicolumn{4}{|c|}{ Visual analogue scale } \\
\hline Day & $60(43-73)$ & $18(12-53)$ & 0.005 \\
\hline Night & $20(3-46)$ & $7(2-27)$ & 0.064 \\
\hline
\end{tabular}

daytime and 72.2\% (IQR 28.6-90.1) for overnight (fig 1, table 2). A paired $t$ test of the log transformed cough rates showed the fall in cough rate to be statistically significant for daytime ( $\mathrm{p}<0.001$, mean difference 2.29 -fold (95\% CI 2.29 to $3.26))$ and night time $(\mathrm{p}=0.049$, mean difference 2.51 -fold (95\% CI 1.02 to 6.31$)$ ). Two patients had an increase in the daytime cough rate and four had an increase in the nocturnal cough rate; however, the total cough rates (cumulative day and night) fell in all patients. These patients did not have a different clinical outcome to the remainder of the group. Patients had a wide range of cough rates and there was a significant diurnal variation in cough rate on both study days $(\mathrm{p}<0.001$ admission, $\mathrm{p}=0.002$ discharge; table 2$)$.

The temporal change in cough seconds over each recording period is shown in fig 2 . The median cough rate at each time point is higher and more variable on admission than at discharge during the day. Coughing virtually stops overnight. When $\log _{10}$ cough epochs are compared with $\log _{10}$ cough seconds, there are significant linear relationships (admission $r=0.92, \mathrm{p}<0.001$; discharge $r=0.92, \mathrm{p}<0.001$ ). However, the length of cough epochs (to the nearest second) significantly reduced with treatment (median (IQR) 2 seconds (1-2) on admission; l second ( $1-1)$ on discharge, $\mathrm{p}=0.009)$.
Statistical analysis (linear regression) did not find a significant difference between the relationships between cough seconds and cough epochs over the short period analysed.

Relationships between coughs and clinical measures There were improvements in lung function, a decrease in daytime sputum weight, and a decrease in CRP levels with treatment of the infective exacerbation (table 2). Multivariate regression analyses were performed to examine the predictive

Table 3 Correlations between changes in subjective estimates of cough frequency (cough scores and visual analogue scale, VAS) and time spent coughing during the day and night

\begin{tabular}{lll}
\hline & $\begin{array}{l}\text { Change in day cough } \\
\text { rate }\end{array}$ & $\begin{array}{l}\text { Change in night cough } \\
\text { rate }\end{array}$ \\
\hline $\begin{array}{l}\text { Change in cough } \\
\text { score }\end{array}$ & $r=0.34, p=0.204$ & $r=0.19, p=0.510$ \\
Change in VAS & $r=0.47, p=0.070$ & $r=0.81, p=0.001$ \\
\hline
\end{tabular}

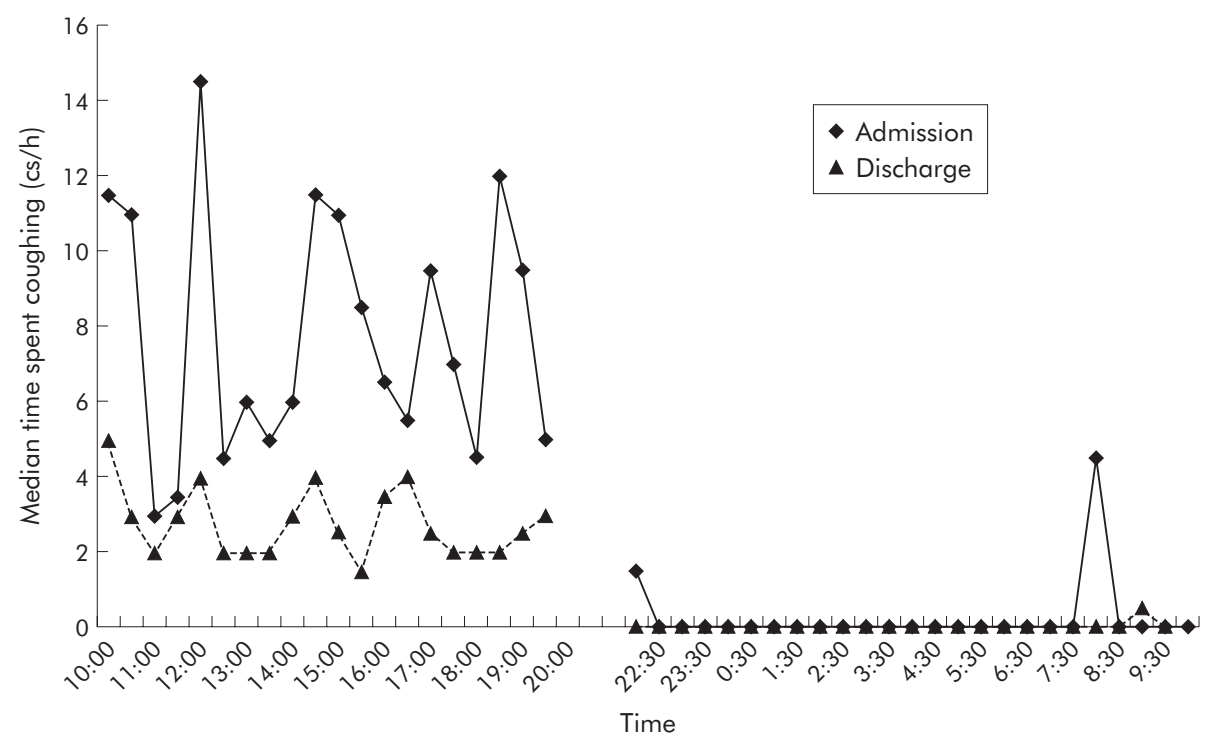

Figure 2 Temporal distribution of coughs during daytime and overnight on admission and before discharge (median time spent coughing per 30 minute period). 
value of $\mathrm{FEV}_{1}$, sputum weight, and CRP for time spent coughing during the day and night on admission and on discharge. Variables were again $\log _{10}$ transformed for analysis to allow the use of parametric statistics. $\mathrm{FEV}_{1}$ $(\mathrm{B}=-0.321(95 \% \mathrm{CI}-0.459$ to -0.184$), \mathrm{p}<0.001)$ and $\mathrm{CRP}$ $(\mathrm{B}=0.006 \quad(95 \% \quad \mathrm{CI} \quad 0.001$ to 0.012$), \mathrm{p}=0.026)$ were significant predictors of overnight cough frequency on admission. Together they accounted for $74.4 \%$ of the variance in time spent coughing overnight (adjusted $R^{2}=0.744$ ). An increase in $\mathrm{FEV}_{1}$ of $100 \mathrm{ml}$ was associated with a $4.7 \%$ fall in cough frequency and an increase in CRP of 1 unit was associated with a $1.4 \%$ increase in cough frequency.

Statistically significant models could not be produced with these parameters for daytime cough frequency on admission or night cough frequency on discharge. Daytime cough frequency on discharge was predicted by sputum volume alone $(\mathrm{B}=0.411 \quad(95 \%$ CI 0.144 to 0.678$), \mathrm{p}=0.005)$. An increase in sputum weight of $1 \mathrm{~g}$ was associated with a $4.0 \%$ increase in daytime cough frequency.

\section{Subjective assessment of cough}

Subjective assessments of cough fell from admission to discharge in keeping with the change in time spent coughing (table 2). Daytime scores were higher than night time scores before and after treatment. The relationships between the change in subjective cough scores, visual analogue scales, and the change in time spent coughing are shown in table 3. The changes in cough scores were not significantly correlated with objective coughing for the day or the night. Visual analogue scales, however, were strongly and significantly correlated with objective coughing overnight and showed a trend for a moderate correlation for change in daytime objective coughing.

\section{DISCUSSION}

This is the first study to quantify the effects of inpatient treatment on coughing in adults with an infective exacerbation of CF. We have shown that the time spent coughing by these patients significantly falls with inpatient treatment including intravenous antibiotics and chest physiotherapy.

The precise mechanisms by which coughing occurs in CF are not understood. The most obvious candidates are airway inflammation related to chronic infection and the accumulation of purulent airway secretions; extrapulmonary diseases such as sinusitis and gastro-oesophageal reflux could also contribute. ${ }^{12}{ }^{13}$ In subjects suffering from chronic dry cough, increased inflammatory mediators have been found in induced sputum ${ }^{14}{ }^{15}$ and have been implicated in increasing the sensitivity of the cough reflex. ${ }^{16}$ In children with stable $\mathrm{CF}$, however, the cough reflex sensitivity to inhaled tussigenic agents has been shown to be similar to or less sensitive than that in healthy controls. ${ }^{17}$ This may not be the case in adults where pulmonary disease has progressed further. Whether cough reflex sensitivity increases during an exacerbation has not been studied in CF, but adults with non-CF bronchiectasis do have an increased cough reflex during exacerbations. ${ }^{18}$

Our secondary outcome measures suggest that overnight cough frequency is influenced by $\mathrm{FEV}_{1}$ and CRP levels in patients with an exacerbation, implying that cough may be driven mainly by airway inflammation. Sputum weight did not influence cough frequency while the subjects were unwell, but after treatment the daytime cough rates increased with increasing sputum weight.

These were not the primary end points in this study and so must be interpreted cautiously; however, as CRP levels and $\mathrm{FEV}_{1}$ explain such a large proportion of the variation in cough rates, this finding is difficult to ignore. Overnight, subjects are less likely to be exposed to environmental irritants or changes in temperature that may independently provoke coughing. The coughing that occurs overnight with exacerbations may therefore be a better reflection of airway inflammation than daytime coughing. Furthermore, factors which then adversely affect the ability of subjects to clear secretions when coughing (such as impaired $\mathrm{FEV}_{1}$ ) would also be expected to influence the frequency and pattern of the cough. In this respect, sputum viscosity, airway instability, and the degree of airflow obstruction are all likely to be important. ${ }^{19}$

Before discharge the only statistically significant predictor of cough rate was daytime sputum volume. Subjects do not always expectorate, so sputum volumes are known to be poor markers of changes in disease and, in addition, this finding was limited to daytime cough rate. A similar effect would be difficult to identify overnight as the majority of overnight sputum volumes fell to zero after treatment.

Although adults with CF cough much more during the day than at night, they are poor at subjectively estimating the severity of their daytime cough. Subjects may become so accustomed to high daytime cough rates that they do not perceive coughing as interfering with their daily activities and are not able to estimate changes well. It is also possible that, when scoring cough severity, subjects take into account other factors not measured in this study such as intensity of the cough. Visual analogue scales are clearly superior to cough scores for subjective estimation of changes in cough frequency in adults with CF.

Discrepancies between subjective and objective assessments of cough frequency have been reported in other conditions where cough is a predominant symptom. In a group of adults with cough variant asthma there was no relationship between objective and subjective measures. ${ }^{7}$ Poor agreement for reporting of nocturnal cough compared with tape recordings was also found in a group of children with asthma. ${ }^{20}$ However, adults with seasonal allergic rhinitis had a significant moderate correlation between objective and subjective cough frequency. ${ }^{21}$ In children with chronic recurrent cough there was an excellent correlation between objective cough frequency and diary card visual analogue scales scored by the children and their parents. ${ }^{22}$ The usefulness of subjective measures of cough seems to vary with subject groups and the tools employed.

The only significant correlation for subjective measures in this study occurred between changes in time spent coughing overnight and visual analogue scales overnight. We speculate that sleep disruption may be the explanation for this. As subjects are most aware of overnight coughing, clinicians should pay particular attention to reporting of worsening overnight coughing as an indicator of an exacerbation.

Our findings of a substantial fall in the cough rate in adults treated for an exacerbation of CF are in contrast to a similar study in children with CF. In that study the cough was identified from sound and EMG signals and cough epochs (that is, bursts of coughing) were counted. ${ }^{4}$ In children with $\mathrm{CF}$ cough and sputum production may be less responsive to treatment. We found that the length of cough epochs significantly shortened from admission to discharge. A device counting only epochs could not appreciate this change and hence cough epochs may be an inferior method for quantifying cough. Measuring epoch frequency may underestimate the reduction in coughing with treatment in CF. However, we could not demonstrate a significant difference between the measures analysed over a 3 hour period.

The main limitation of this study is that available digital recording equipment does not currently allow continuous 24 hour sound recordings of adequate quality. Rapid improvements in storage capacity and battery life should make this possible soon. Manual counting to quantify cough 
is time consuming and hence studies are restricted to relatively small numbers of subjects; however, algorithms to identify cough automatically are showing promise.

In conclusion, we have used an accurate although labour intensive method for objectively quantifying cough in adults with CF. We were able to quantify the change in cough frequency associated with treatment of an acute exacerbation. Cough rates during an exacerbation may be more influenced by airway inflammation and $\mathrm{FEV}_{1}$ than sputum volume but the relationships may be reversed after treatment. An accurate 24 hour automated cough monitor would greatly improve the evaluation of the burden of cough in all respiratory diseases.

\section{ACKNOWLEDGEMENTS}

The authors thank all the patients for taking part, the physiotherapists for their help in patient recruitment, and Mr Kevin McGuiness for maintaining the recording equipment.

\section{Authors' affiliations}

J A Smith, E C Owen, A Woodcock, North West Lung Centre, South Manchester University Hospitals Trust, Manchester, UK

A M Jones, M E Dodd, A K Webb, Manchester Adult Cystic Fibrosis Centre, South Manchester University Hospitals Trust, Manchester, UK Funding: North West Lung Centre Research Fund.

Competing interests: none.

\section{REFERENCES}

1 Ramsey BW, Boat TF. Outcome measures for clinical trials in cystic fibrosis. Summary of a Cystic Fibrosis Foundation Consensus Conference. J Pediatr 1994; 124:177-92.

2 Rosenfeld M, Emerson J, Williams-Warren J, et al. Defining a pulmonary exacerbation in cystic fibrosis. J Pediatr 2001;139:359-65.

3 Dakin C, Henry RL, Field P, et al. Defining an exacerbation of pulmonary disease in cystic fibrosis. Pediatr Pulmonol 2001;31:436-42.
4 Hamutcu R, Francis J, Karakoc F, et al. Objective monitoring of cough in children with cystic fibrosis. Pediatr Pulmonol 2002;34:331-5.

5 Smith J, Halls L, Hiew Y, et al. Cough in cystic fibrosis. Am J Respir Crit Care Med 2001;165:A287.

6 Smith JA, Hiew Y, Cheetham BMG, et al. Cough recognition: video vs. sound recording in the laboratory. Am J Respir Crit Care Med 2002; 165:A382.

7 Hsu JY, Stone RA, Logan-Sinclair RB, et al. Coughing frequency in patients with persistent cough: assessment using a 24 hour ambulatory recorder. Eur Respir J 1994;7:1246-53.

8 Power JT, Stewart IC, Connaughton JJ, et al. Nocturnal cough in patients with chronic bronchitis and emphysema. Am Rev Respir Dis 1984;130:999-1001.

9 Munyard P, Bush A. How much coughing is normal? Arch Dis Child 1996;74:531-4

10 Munyard P, Busst C, Logan-Sinclair R, et al. A new device for ambulatory cough recording. Pediatr Pulmonol 1994;18:178-86.

11 Bland JM, Altman DG. Statistical methods for assessing agreement between two methods of clinical measurement. Lancet 1986;1:307-10.

12 Brodzicki J, Trawinska-Bartnicka M, Korzon M. Frequency, consequences and pharmacological treatment of gastroesophageal reflux in children with cystic fibrosis. Med Sci Monit 2002;8:CR529-37.

13 Ledson MJ, Tran J, Walshaw MJ. Prevalence and mechanisms of gastrooesophageal reflux in adult cystic fibrosis patients. J R Soc Med 1998;91:7-9.

14 Chaudhuri R, McMahon AD, Thomson $\amalg$, et al. Effect of inhaled corticosteroids on symptom severity and sputum mediator levels in chronic persistent cough. J Allergy Clin Immunol 2004;113:1063-70.

15 Birring SS, Parker D, Brightling CE, et al. Induced sputum inflammatory mediator concentrations in chronic cough. Am J Respir Crit Care Med 2004; 169:15-9.

16 Choudry NB, Fuller RW, Pride NB. Sensitivity of the human cough reflex: effect of inflammatory mediators prostaglandin E2, bradykinin, and histamine. Am Rev Respir Dis 1989:140:137-41.

17 Chang AB, Phelan PD, Sawyer SM, et al. Cough sensitivity in children with asthma, recurrent cough, and cystic fibrosis. Arch Dis Child 1997;77:331-4.

18 Choudry NB, Fuller RW. Sensitivity of the cough reflex in patients with chronic cough. Eur Respir J 1992;5:296-300.

19 Shah PL, Scott SF, Knight RA, et al. In vivo effects of recombinant human DNase I on sputum in patients with cystic fibrosis. Thorax 1996;51:119-25.

20 Falconer A, Oldman C, Helms P. Poor agreement between reported and recorded nocturnal cough in asthma. Pediatr Pulmonol 1993;15:209-11.

21 Krahnke J, Gentile D, Angelini B, et al. Comparison of objective and subjective measurements of cough frequency in patients with seasonal allergic rhinitis. Ann Allergy Asthma Immunol 2004;93:259-64.

22 Chang $A B$, Phelan PD, Robertson CF, et al. Frequency and perception of cough severity. J Paediatr Child Health 2001;37:142-5. 